Test yourself in eczema in primary immunodeficiency

\title{
Nesrine Radwan
}

Lecturer of Pediatrics, Ain Shams University

Choose only one answer:

1. Which of the following is characteristic of WAS?
a) Eczema presenting in older age
b) Thrombocytopenia
c) Low B cell
d) Normal T cell
e) All of the above

2. Immunological features of Omenn syndrome include(s):
a) Normal Immunoglobulin level
b) Eosinophilia
c) Low $T$ cell number
d) Good response to vaccination
e) Abnormal $\mathrm{T}$ cell proliferation

5. A patient aged 3 years, presenting with eczema, recurrent sino-pulmonary infections and viral infections, neutropenia. He had normal immunoglobulin level. He had mild CD4+ lymphopenia. He had mild pulmonary stenosis which was accidentally discovered. His EBV PCR was persistently positive for the last 6 months. What is your provisional diagnosis?
a) DOCK8 deficiency
b) $\mathrm{AD}$ hyperimmunoglobulin $\mathrm{E}$
c) Wiscott Aldrich Syndrome
d) Mammalian sterile 20-like 1(MST-1)
e) Shwachman- Diamond syndrome

(Answers on page 49)

3. To differentiate between eczema in an atopic patient and a patient with AD hyperimmunoglobulin $\mathrm{E}$ syndrome all are true EXCEPT:
a) Site of eczema
b) Response to treatment
c) Presence of infection of top
d) Elevated IgE > $1000 \mathrm{IU} / \mathrm{L}$
e) Age of onset

4. Characteristic features in DOCK8 deficiency includes all EXCEPT:
a) Presence of eczema
b) Recurrent sinopulmonary infections
c) Recurrent viral infections
d) Low CD4+ cells and CD8+
e) Coarse features 